\title{
Probing the decay mechanism of hot nuclei by Coulomb chronometry
}

\author{
D. Gruyer, E. Bonnet, A. Chbihi and J. D. Frankland \\ for the INDRA COLLABORATION
}

GANIL, CEA-DSM/CNRS-IN2P3, Caen, France

\begin{abstract}
In this contribution, we propose a new Coulomb chronometer suitable for three-fragment exit channels. We use this chronometer to extract the evolution of the fragment emission time in ${ }^{129} \mathrm{Xe}+{ }^{c a t} \mathrm{Sn}$ central collisions from 12 to $25 \mathrm{MeV} / \mathrm{A}$ bombarding energy. The involved time scale becomes compatible with simultaneous threefragment break-up above $E^{*}=4.0 \pm 0.5 \mathrm{MeV} / \mathrm{A}$, which can be interpreted as the energy required for the onset of multifragmentation.
\end{abstract}

\section{Introduction}

Recent data on ${ }^{129} \mathrm{Xe}+{ }^{c a t} \mathrm{Sn}$ central collisions [1] show that at $8 \mathrm{MeV} / \mathrm{A}$ bombarding energy, almost all the reaction cross section is composed of events with two heavy fragments in the exit channel (see Fig. 1(a)). Above $12 \mathrm{MeV} / \mathrm{A}$ bombarding energy, the three-fragment exit channel becomes significant, overcoming the two-fragment production rate above $18 \mathrm{MeV} / \mathrm{A}$. The decay mechanism responsible for these three-fragment events is not well established: Is it the continuation of low energy fission or the precursor of high energy simultaneous fragmentation? To answer this question, a dynamical characterization of the decay mechanism is needed. In particular, the estimation of the involved time scales may allow to disentangle sequences of two binary fission from simultaneous three-fragment break-up.

In this contribution, we propose a new Coulomb chronometer suitable for three-fragment exit channels. We use this chronometer to extract the evolution of the fragment emission time in ${ }^{129} \mathrm{Xe}+{ }^{\text {cat }} \mathrm{Sn}$ central collisions from 12 to $25 \mathrm{MeV} / \mathrm{A}$ bombarding energy. 


\section{Experimental analysis}

Collisions of ${ }^{129} \mathrm{Xe}+{ }^{\text {cat }} \mathrm{Sn}$ at $12,15,18,20$, and $25 \mathrm{MeV} / \mathrm{A}$ were measured using the INDRA $4 \pi$ charged product array [2] at the GANIL accelerator facility. In this analysis, we considered only fusion-like events leading to three heavy fragments $(Z>10)$ in the exit channel. Fusion-like events were selected by requiring at least $90 \%$ of the total charge of the system to be detected.

We start from the hypothesis that fragments are produced sequentially. If two successive splittings occur, three possible sequences of splittings have to be considered.
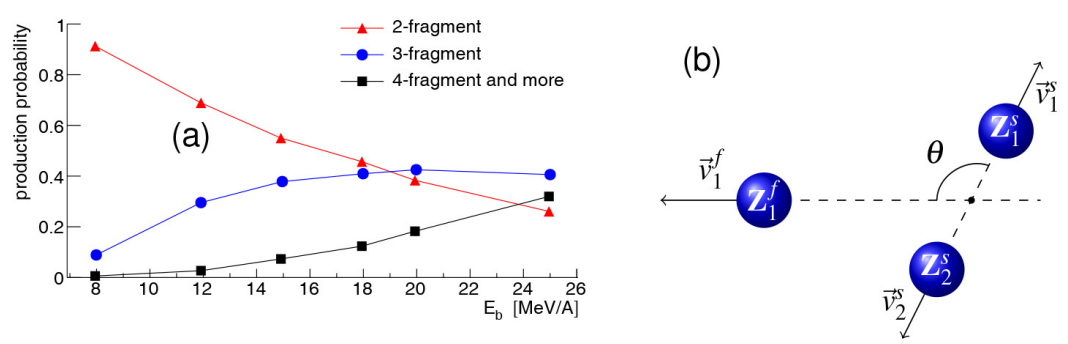

Figure 1: (color online). (a) Evolution of different exit channel probabilities as a function of the beam energy for ${ }^{129} \mathrm{Xe}+{ }^{\text {cat }}$ Sn central collisions. (b) Definition of the relevant kinematic observables for the three-fragment exit channel, in the rest frame of the intermediate system $\mathrm{Z}_{2}^{f}$.

To identify the sequence of splittings event by event, we compare the relative velocity between each pair of fragments with that expected for fission, taken from the Viola systematics [3,4]. The pair with the most Violalike relative velocity is considered to have been produced during the second splitting. We can therefore trivially deduce that the remaining fragment was emitted first. Once the sequence of splittings is known event by event, fragments can be sorted according to their order of production. Let us now call $\mathrm{Z}_{1}^{f}$ and $\mathrm{Z}_{2}^{f}$, the two nuclei coming from the first splitting. The fragment $\mathrm{Z}_{2}^{f}$ breaks in $\mathrm{Z}_{1}^{s}$ and $\mathrm{Z}_{2}^{s}$ during the second step (see Fig. 1).

To estimate the mean inter-splitting time, we used the correlation between the inter-splitting angle $\theta$ and the relative velocity of the second splitting: $v_{12}^{s}=\left\|\vec{v}_{1}^{s}-\vec{v}_{2}^{s}\right\|$ (see Fig.2(a)). These correlations present a maximum at $\theta \sim 90$, which is more pronounced as the beam energy increases. We quantify this effect by the Coulomb distortion parameter $\delta v=v_{12}^{s}(90)-v_{12}^{s}(0)$ which increases with increasing beam energy (Fig.2(b)), indicating that the second splitting occured closer and closer to the first emitted fragment. To 
translate $\delta v$ in terms of inter-splitting time $\delta t$, we performed Coulomb trajectory calculations for point charges, which simulate sequential break-ups using experimental mean charges. Finally, we obtained the evolution of the inter-splitting time as a function of the beam energy (Fig.3).
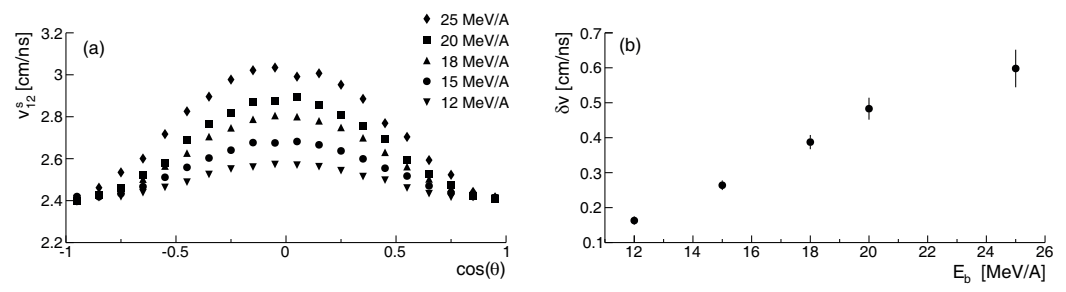

Figure 2: (a) Correlation between the inter-splitting angle $\theta$ and the relative velocity of the second splitting $v_{12}^{s}$, vertical error bars are smaller than the size of the points; (b) evolution of the Coulomb distortion parameter $\delta v$ as a function of the beam energy for ${ }^{129} \mathrm{Xe}+{ }^{\text {cat }} \mathrm{Sn}$ central collisions.

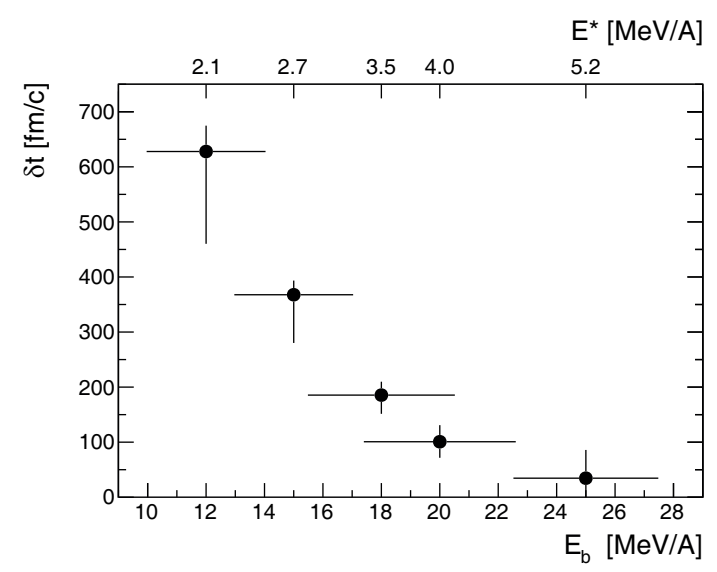

Figure 3: Evolution of the mean inter-splitting time $\delta t$ as a function of the beam energy (lower scale) and the estimated excitation energy of the incomplete fusion source (upper scale) produced in ${ }^{129} \mathrm{Xe}+{ }^{c a t} \mathrm{Sn}$ central collisions. Horizontal error bars refer to the upper scale.

\section{Discussion}

A clear decrease of the inter-splitting time with increasing beam energy is observed in Fig.3. At $12 \mathrm{MeV} / \mathrm{A}$, the inter-splitting time $\delta t$ is of the order of $600 \mathrm{fm} / \mathrm{c}$. It shows that, for the lower beam energies, fragments arise from 
two successive splittings, validating our starting hypothesis. As the beam energy increases from $12 \mathrm{MeV} / \mathrm{A}$ to $20 \mathrm{MeV} / \mathrm{A}$, $\delta t$ decreases monotonically from $600 \mathrm{fm} / \mathrm{c}$ to about $100 \mathrm{fm} / \mathrm{c}$. Above $20 \mathrm{MeV} / \mathrm{A}, \delta t$ becomes compatible with zero. Our trajectory calculations show that, below $\delta t \sim 100 \mathrm{fm} / \mathrm{c}$, fragment emissions can not be treated independently, and it is no longer meaningful to speak of a sequential process. Therefore, Fig. 3 shows that the three-fragment exit channel is compatible with successive binary splittings on shorter and shorter time scales, becoming indistinguishable from simultaneous three-fragment break-up at bombarding energies above $20 \mathrm{MeV} / \mathrm{A}$, which correspond to $E^{*} \sim 4 \pm 0.5 \mathrm{MeV} / \mathrm{A}$.

\section{Conclusion}

In summary, we proposed a new chronometer which takes advantage of Coulomb proximity effects observed in the three-fragment final state. This is made possible thanks to the highly exclusive measurement performed with INDRA. We have shown that these fragments arise from successive binary splittings occurring on shorter and shorter time scales.

The involved time scale becomes compatible with simultaneous threefragment break-up above $E^{*}=4.0 \pm 0.5 \mathrm{MeV} / \mathrm{A}$, which can be interpreted as the signature of the onset of multifragmentation.

\section{References}

[1] A. Chbihi, L. Manduci et al., J. Phys.: Conf. Ser. 420012099 (2013).

[2] J. Pouthas, B. Borderie et al., Nucl. Instrum. Methods Phys. Res., Sect A 357418 (1995).

[3] V.E. Viola, K. Kwiatkowski and M. Walker, Phys. Rev. C: Nucl. Phys, 311550 (1985).

[4] D. Hinde, J. Leigh et al., Nucl. Phys. A 472318 (1987). 